\title{
Study of Elongation and Temperature Effects on Nanocomposite Based on Elastic Fiber ${ }^{\dagger}$
}

\author{
Renato Torres, Adytia Jalasutram, Abderrahmane Benchirouf, Christian Müller and \\ Olfa Kanoun * \\ Technische Universität Chemnitz, 09111 Chemnitz, Germany; \\ renato-da-veiga.torres@s2015.tu-chemnitz.de (R.T.); aditya.jalasutram@s2015.tu-chemnitz.de (A.J.); \\ abderrahmane.benchirouf@etit.tu-chemnitz.de (A.B.); christian.mueller@etit.tu-chemnitz.de (C.M.) \\ * Correspondence: mst@etit.tu-chemnitz.de; olfa.kanoun@etit.tu-chemnitz.de; Tel.: +49-(0)371-531-24480 \\ † Presented at the Eurosensors 2017 Conference, Paris, France, 3-6 September, 2017.
}

Published: 7 August 2017

\begin{abstract}
Stretchable sensors are increasingly demanded in the fields of smart textiles for sports, military and medical industry. In this work, we propose novel elongation sensitive elastic fibers made up of Multi-Walled Carbon Nanotubes (MWCNT), Polyester and Polyurethane. The entire fiber is encased with natural rubber (NR) for protection against mechanical, electrical and humidity external influences. With this fiber, it is possible to reach elongations above $100 \%$. It can be fabricated in different sizes according to application requirements. The relationship between fiber resistance and its physical modifications was measured at different elongations and temperatures. The experimental results show a high sensitivity and a stable temperature behavior.
\end{abstract}

Keywords: stretchable sensor; strain sensor; elongation sensor; carbon nanotubes; smart textiles

\section{Introduction}

The demand for smart textiles is growing up due to different trends, such as wearable computing, smart health and integrated lightweight structure. Especially for sensors on the periphery of human body, skin compatibility, low cost, simple manufacturing and compatibility with common textile materials are strongly required. For these reasons, we propose to use Polyurethane twisted in polyester fiber [1] as high flexibility basis fiber material and then coat it with a film of carbon nanotubes, which has a high sensitivity to strain and elongation. As coating material, we propose to use a natural rubber, which has a high flexibility and outstanding encapsulation and skin protection properties.

\section{Materials and Methods}

The demand for smart textiles is growing up due to different trends, such as wearable computing, smart health and integrated lightweight structure. Especially for sensors on the periphery of human body, skin compatibility, low cost, simple manufacturing and compatibility with common textile materials are strongly required. For these reasons, we propose to use Polyurethane twisted in polyester fiber [1] as high flexibility basis fiber material and then coat it with a film of carbon nanotubes, which has a high sensitivity to strain and elongation. As coating material, we propose to use a natural rubber, which has a high flexibility and outstanding encapsulation and skin protection properties.

The highly concentrated dispersion of MWCNTs is realized with a water solution of Sodium dodecylbenzene sulfonate (SDBS) treated by magnetic stirring for $20 \mathrm{~h}$ at room temperature. Samples of Polyurethane fiber twisted in polyester fiber are then placed into the MWCNT solution for $30 \mathrm{~min}$. 
By drying process in oven at $60{ }^{\circ} \mathrm{C}$ for $30 \mathrm{~min}$ the MWCNT is fixed on the surface [2]. The illustrated fabricated fibers (Figure 1b) are taken out of the oven then dipped inside the NR and dried for $15 \mathrm{~h}$.

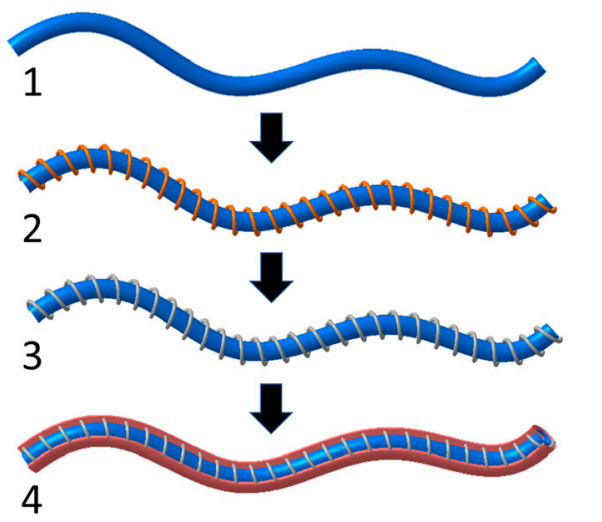

(a)

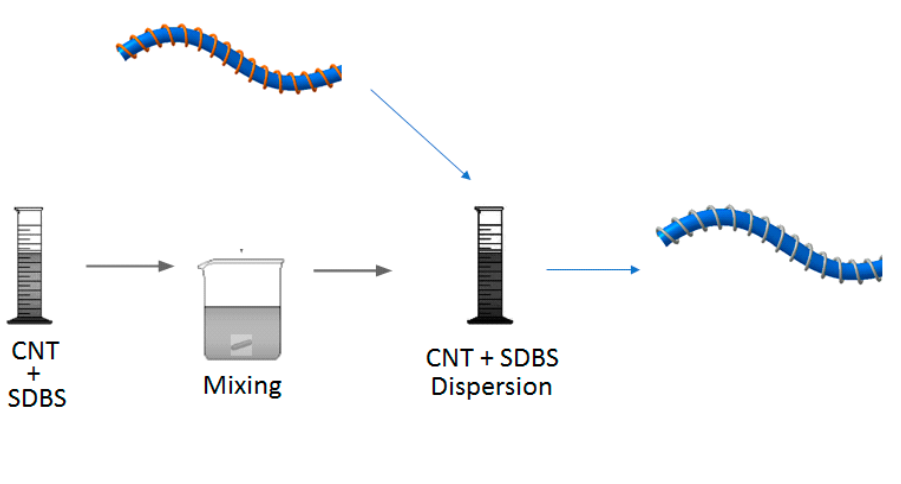

(b)

Figure 1. Obtainment of filament based sensor: (a) Fiber based sensor synthesis stages: (a.1) Polyurethane core fiber, (a.2) Polyurethane core fiber + Polyester fibers, (a.3) Polyurethane core fiber + Polyester fibers coated with MWCNT (a.4) Polyurethane core fiber + Polyester fibers coated with MWCNT, encased (coated) with Latex; (b) Laboratory sequence for solution and coating making.

\section{Results}

\subsection{Microscopical Characterization}

Optical images were obtained using Keyence VHX Digital Microscope showing polyester fibers after coating process under MWCNT solution. It is possible to observe in Figure $2 a$ that MWCNT solution is fixed only over polyester fibers, middle polyurethane core is not affected by MWCNT solution.

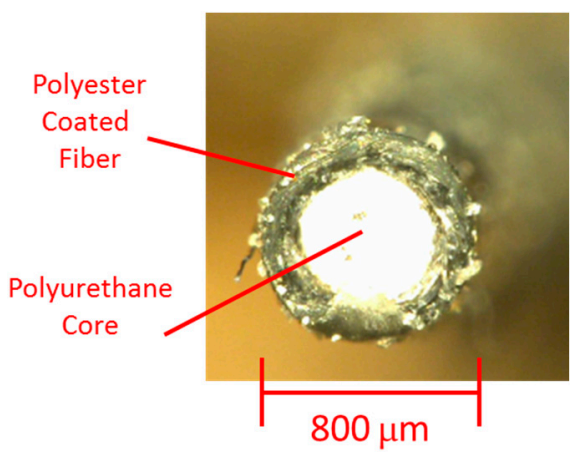

(a)

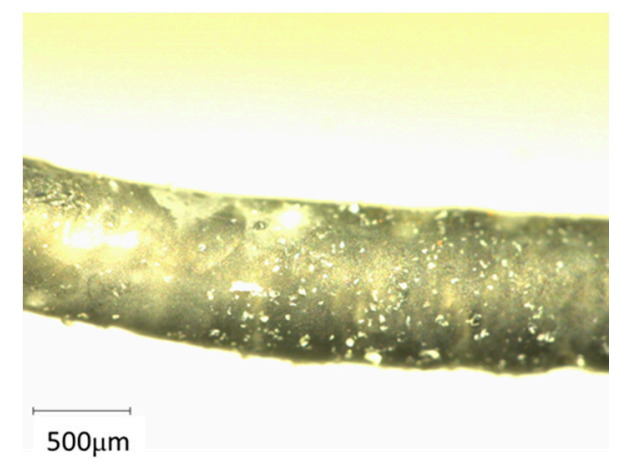

(b)

Figure 2. Microscopic images of obtained fibers after coating and sealing process: (a) Cross section showing coated polyester fibers rounding a polyurethane core; (b) Longitudinal image showing effective latex enclosing.

In Figure $2 \mathrm{~b}$ is possible to check that all filament is sealed by a latex layer, this layer is added to avoid humidity interference, add mechanical, friction and aging protection.

\subsection{Electrical Characterization}

Each sample has a length of $10 \mathrm{~cm}$ and connected with copper wires in its extremities to enable electrical contacting (Figure 3d). The initial resistance of the samples is around $\mathrm{R}_{0}=1.2 \mathrm{M} \Omega$.

For measurement of electrical resistance under tensile strain, fibers were fixed on one end using a clamp. On the other side, the free end of the sample was automatically elongated by an actuator in 
the direction of its length and held using another clamp as shown in Figure 3. Its length was incremented in steps of $1 \mathrm{~mm}$. For each step, its resistance was measured during elongation (loading) and relaxation (unloading). During the experiment, up to $25 \%$ of elongation was applied on the sample as shown in Figure 3a,b. Different samples were evaluated to check the reproducibility of the manufacturing process. They show a similar non-linear behavior with a high sensitivity at the low elongation range up to $5 \mathrm{~mm}$ which is approximately $160 \mathrm{in} \mathrm{k} \Omega / \mathrm{mm}$. At higher elongation between 15 and $25 \mathrm{~mm}$, the sensitivity is approximately $40 \mathrm{k} \Omega / \mathrm{mm}$.

For measurement of electrical resistance under temperature change, samples were evaluated using a climate chamber and the resistance was recorded under temperature variation from $-10{ }^{\circ} \mathrm{C}$ to $60{ }^{\circ} \mathrm{C}$. The fibers show a stable temperature behavior. During the experiment, the relative change in the resistance reach up to $18 \%$ of initial resistance as was shown in Figure 3c.

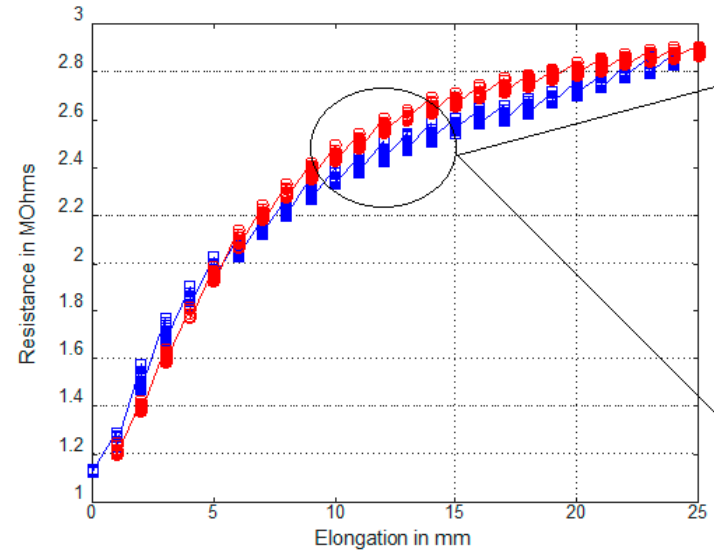

(a)

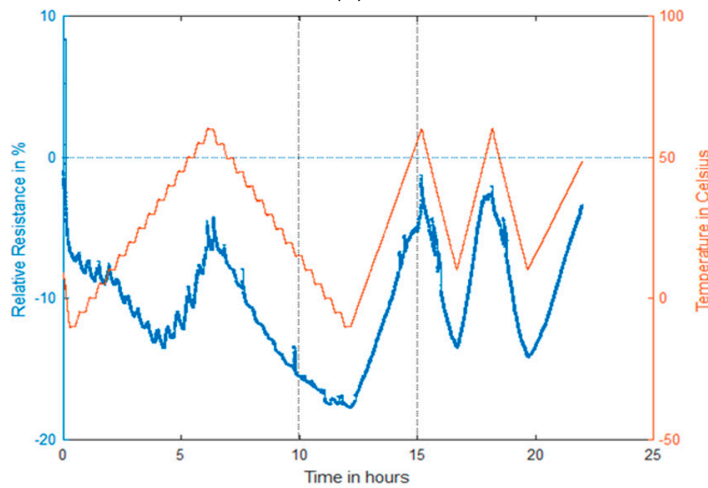

(c)

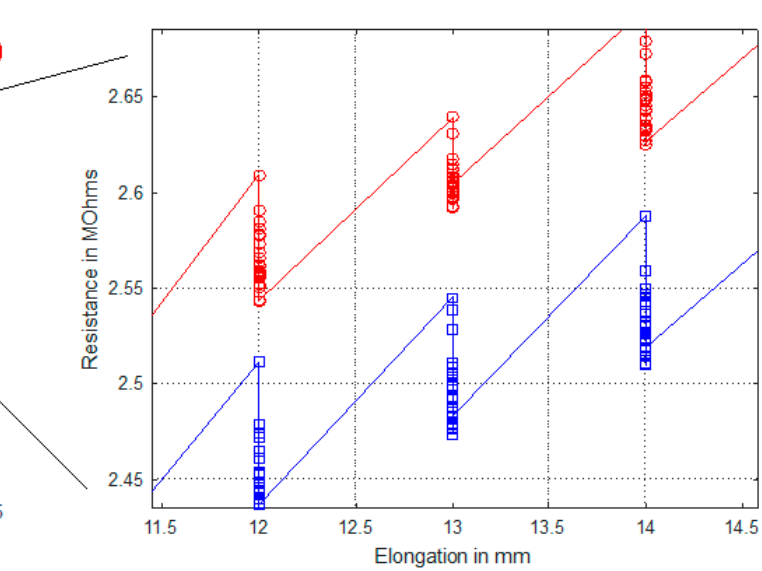

(b)

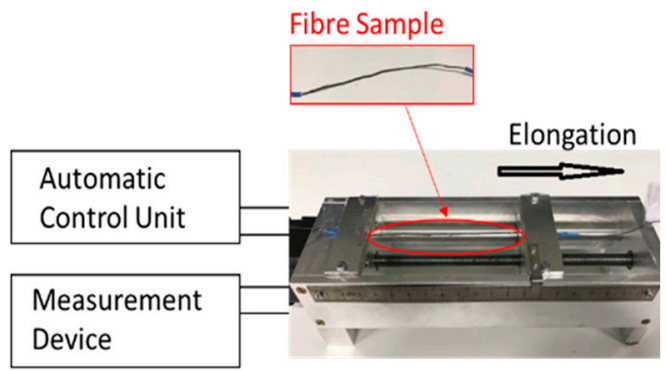

(d)

Figure 3. (a) Measured resistance values for elongation-load in blue and unload in red; (b) Zoomed resistance values at $13 \mathrm{~mm}$; (c) Relative change of resistance with temperature-relative resistance in blue and temperature in red; (d) Test setup for characterization of the sensitive fiber.

\section{Conclusions}

This work shows possibilities for flexible sensor development based on fibers. These fibers show electrical sensitivity $40 \mathrm{k} \Omega / \mathrm{mm}$ in high elongations and higher in reduced range. This behavior is obtained after simple treatment, encouraging development of complete elongation or displacement sensors based on flexible materials. Temperature sensitivity also was detected, providing opportunity to use as rough temperature sensor or demonstrating that is necessary add compensation or correction stage to avoid its interference. SEM images for further characterization, adsorption calculation, improvements in hysteresis modeling/reduction and execution of range/endurance tests are required to candidate this approach as future elongation/strain sensor. 
Acknowledgments: We acknowledge Institute of Physics of Technische Universität Chemnitz for providing all facilities in order to obtain microscopical images. Part of this work is funded by the Brazilian CSF (Ciencia sem Fronteiras) program from CAPES/CNPq.

Conflicts of Interest: The authors declare no conflict of interest.

\section{References}

1. Sun, J.; Huang, Y.; Fu, C.; Wang, Z.; Huang, Y.; Zhu, M.; Zhi, C.; Hu, H., High-performance stretchable yarn supercapacitor based on PPy@CNTs@urethane elastic fiber core spun yarn. Nano Energy 2016, 27, 230-237.

2. Jost, K.; Durkin, D.P.; Haverhals, L.M.; Brown, E.K.; Langenstein, M.; de Long, H.C.; Trulove, P.C.; Gogotsi, Y.; Dion, G. Natural Fiber Welded Electrode Yarns for Knittable Textile Supercapacitors. Adv. Energy Mater. 2015, 5, 1401286.

(C) 2017 by the authors. Licensee MDPI, Basel, Switzerland. This article is an open access article distributed under the terms and conditions of the Creative Commons Attribution (CC BY) license (http://creativecommons.org/licenses/by/4.0/). 